\title{
Interpolation Spaces and Energy Quantization for Yang-Mills Fields
}

\author{
TRISTAN RIVIÈRE
}

\begin{abstract}
In [19] G. Tian proved that the defect measure of a weakly converging sequence of Yang-Mills Fields on a riemannian manifold $(M, g)$ of dimension $n(n \geq 4)$ is carried by a $n-4$-rectifiable subset $S$ of $M$. In the present paper we complete the picture of weakly converging sequences of YM Fields by proving that, in 4 dimension, the defect measure is quantized: at any point of $S$ it is the sum of $L^{2}$ energies of YM fields on $S^{4}$. This result is extended to any dimension under some additional assumption on the $W^{2,1}$ norm of the curvature. In the last part we study non-linearities issued from curvatures in general and we prove that strong convergence in $W^{1, n / 2}$ of Coulomb gauges of connections over a $n$-dimensional manifold preserves the topology of the bundle at the limit.
\end{abstract}

\section{Introduction.}

The first step in the program of building up a Gauge theory in higher dimension (higher than the conformal one) proposed by S.K. Donaldson and R.P. Thomas is to give a precise picture of the non-compactness in $L^{2}$ of Yang-Mills fields on a manifold $M$ bellow some energy level.

Let $E$ be a vector bundle over a riemannian manifold $(M, g)$ with structure group a compact Lie Group $G$ (denote by $\mathfrak{G}$ its Lie Algebra). We will adopt the notations of [6] Chapter 2. A connection $A$ on $E$ is characterized by its corresponding covariant derivative $\nabla_{A}$ acting on sections of $E$, $\Omega^{0}(E)$ into sections of $E \otimes T^{*} M$. From $\nabla_{A}$ one may constuct the exterior derivative $d_{A}$ from $E$-valued $p$-forms, $\Omega^{p}(E)$ into $E$-valued $p+1$-forms ([6] p. 35). We will denote by $F_{A}$ the curvature of $A$ which is a two form into the $\mathfrak{G}$-bundle deduced from the principal bundle of $E$ for the adjoint representation $\left(F_{A} \in \Omega^{2}\left(\mathfrak{G}_{E}\right)\right)$. A Yang-Mills Field of $E$ is a critical point of the Yang-Mills action

$$
\int_{M}\left|F_{A}\right|^{2}
$$


where the scalar product on $\Omega^{2}\left(\mathfrak{G}_{E}\right)$ is deduced from the Killing form of $G$ and the metric $g$ on $M$ (see [6]). $F_{A}$ is Yang-Mills if and only if it solves the Euler-Lagrange Equation

$$
d_{A}^{*} F_{A}=0
$$

where $d_{A}^{*}$ is the adjoint of $d_{A}$ for the above mentioned $L^{2}$-scalar product on $\Omega^{*}\left(\mathfrak{G}_{E}\right)$.

In [19] G. Tian studied sequences of smooth solutions of (I.2) having uniformly bounded energy. One among the main results of Tian's paper can be stated in the following way.

Theorem I.1 ([19]). Let $A_{i}$ be a sequence of Yang-Mills connection with uniformly bounded energy, then one may extract a subsequence $A_{i^{\prime}}$ such that, modulo gauge transformation,

$$
F_{A_{i^{\prime}}} \rightarrow F_{A} \quad \text { weakly in } L^{2}
$$

where $F_{A}$ is the curvature of a smooth Yang-Mills connection outside a subset $\Sigma$ of $M$ having finite $n-4$-Haussdorff dimension,

$$
\left|F_{A_{i^{\prime}}}\right|^{2} d V_{g} \longrightarrow\left|F_{A}\right|^{2} d V_{g}+\Theta \mathcal{H}^{n-4}\lfloor S
$$

as Radon measures where $d V_{g}$ denotes the volume form on $(M, g), \mathcal{H}^{n-4}$ is the $n-4$ Hausdorff measure, $S$ is a $n-4$ rectifiable subset of $M$ containing $\Sigma, \mathcal{H}^{n-4}\lfloor S$ is the restriction to $S$ of the Hausdorff measure and $\Theta$ is a mesurable fonction on $S$ admiting a positive lower bound depending only on $n$.

Of course the main difficulty in the above stated result is to establish the rectifiability property of the energy defect measure of the sequence $A_{i^{\prime}}$ : $\mu=\Theta \mathcal{H}^{n-4}\lfloor S$. The proof in [19] follows the approach of F.H. Lin in [9] where the rectifiability of the defect measure of stationary harmonic maps was established.

The main question we adress in the present paper concerns the multiplicity $\Theta$ of the defect measure in Theorem I.1. In the particular case when we are dealing with special YM connections which are $\Omega$-anti-self-dual for some closed $n-4$ form $\Omega$ (see [19]) G. Tian proved that $\Theta(x)$ is an integer, sum of the energies of anti-self-dual 4-dimensional instantons that concentrate at $x$, which means that there is no loss of energy between "bubbles". This phenomenon can be called the no neck property (see discussions about the existence or non existence of necks in bubble trees for harmonic maps and 
"almost"-harmonic maps on surfaces in [5] and [16]). This no neck property was previously established in [6] in dimension 4 for sequences of anti-selfdual Yang-Mills fields and was a important step in the compactification of the moduli space of ASD-connections on a compact 4-manifolds.

Here we will extend this no neck property to sequences of general smooth YM Fields in dimension larger or equal to 4 . We will use the following notation: $\left|\nabla_{A} \nabla_{A} F_{A}\right|=\sqrt{\sum_{i, j}\left|\nabla_{i} \nabla_{j} F_{A}\right|^{2}}$ and $\nabla_{i}$ denotes the covariant derivative induced by $A$ along the $i$-th direction of $\mathbb{R}^{n}$. Our first main result is the following.

Theorem I.2. Let $n \geq 4$ and let $\mu=\Theta S\left\lfloor\mathcal{H}^{n-4}\right.$ be the defect measure of a sequence of Yang-Mills connections given in Theorem I.2, assuming in the case where $n>4$ that $\left\|\nabla_{A} \nabla_{A} F_{A}\right\|_{L^{1}}$ is uniformly bounded, then $\mu$ is quantized:

$$
\text { for } \mathcal{H}^{n-4} \text { a. e. } x \in S \quad \Theta(x)=\sum_{j=1}^{N_{x}}\left\|F_{B_{j}}\right\|_{L^{2}}^{2}
$$

where $N_{x}$ is a positive integer and $B_{j}$ are Yang-Mills connections of $G$ bundles over $S^{4}$ issued from $A_{i^{\prime}}$ and that concentrate at $x$ as $i \rightarrow+\infty$.

Remark I.1. We believe that the assumption $\left\|\nabla_{A} \nabla_{A} F_{A}\right\|_{L^{1}}$ to be uniformly bounded in dimension strictly larger than 4 could be removed from the statement of Theorem I.2 above as it is here for $n=4$.

Remark I.2. The result above is extended to sequences of eventualy singular Yang-Mills Fields that are stationary in [13].

The meaning of issued from $A_{i^{\prime}}$ and that concentrate at $x$ as $i \rightarrow+\infty$ has to be understood in the following way : There are conformal maps $\psi_{j}^{i}$ from $S^{4}$ minus a fixed number of balls shrinking to zero, $\cup_{k} B_{i, j}^{k}$, into $N_{x}$ disjoint subparts of the 4-dimensional plane perpendicular to $T_{x} S$ in $T_{x} M$ such that the pull-back bundle $\psi_{j}^{i *} \exp _{x}^{*} E$ extends to the smooth bundle over $S^{4}$ on which $B_{j}$ is defined, moreover the pull back curvature $\psi_{j}^{i *} \exp ^{*} F_{A_{i^{\prime}}}$ converges strongly in $L^{2}\left(S^{4} \backslash \cup_{k} B_{i, j}^{k}\right)$ to $F_{B_{j}}$ (modulo changes of gauge). $\exp _{x}$ denotes the exponential map from $\left(T_{x} M, g_{x}\right)$ into $(M, g)$.

The above stated quantization result we shall prove bellow is established using technics introduced by the author in collaboration with F.H. Lin while studying Ginzburg-Landau vortices in [10]. Right after this work we realized 
that these techniques could be developped in order to prove the corresponding statement to Theorem I.2 but for harmonic maps between manifolds in [11] (see also [12] for an adaptation of these techniques to parabolic situations). As the main tool we introduced the use of Lorentz Spaces on subspaces having the conformal dimension of the problem. More specificaly the originality of these works was to present a use of the $L^{2, \infty}-L^{2,1}$ duality as the right substitue of the apparently natural $L^{2}-L^{2}$ duality in which we got stucked for quite a long time. This slight shift in duality is also the key point of the analysis we will present bellow. In the last part of the paper we extend the use of Lorentz Spaces to general curvatures of bundles over manifolds. One of the crucial observation is the following (for instance in 4-dimension): assume we are working in a Gauge $A$ which is controled in the $W^{1,2}$-norm (the conformal invariant 1 in 4-dim) from the Peetre-Sobolev embeddings [15] we deduce that $A$ is controled in the Lorentz norm $L^{4,2}$ and from standard properties of products in Lorentz Spaces we obtain that $[A, A]$ is controled in $L^{2,1}$ norm: in other words in the curvature $F_{A}=d A+[A, A]$ we observed that the non-linear part $[A, A]$, which makes the analysis of curvatures usualy quite delicate, is in fact slightly more regular (in $L^{2,1}$ ) than the linear part $d A$ itself which is a-priori only in $L^{2}$. This kind of idea is also adapted to Yang-Mills equations (see Lemma II.3). In the last theorems of the paper we prove that strongly converging sequences of Coulomb gauges in $W^{1, n / 2}$ for a given bundle over a n-manifold preserve the topology of the bundle at the limit. This was well known in $W^{1, p}$ for $p>n / 2$ (see [21]) and is not true anymore in $W^{1, p}$ for $p<n / 2$. These borderline situation is solved by the mean of Brezis-Wainger-Sobolev embeddings for Lorentz Spaces (see $[3])$.

Acknowledgments. The author is very gratefuhl to Fanghua Lin for the exchanges of ideas which have motivated some aspects of this work. The author would like also to express his gratitude to Haïm Brezis and Luc Tartar for their comments and stimulating discussions on Lorentz Spaces.

\section{Proof of Theorem I.2 for $n>4$.}

In order to simplify the presentation of the proof again, since the result is essentially local, we take the basis of $E$ to be the flat unit ball $B^{n}$ of $\mathbb{R}^{n}$.

Let $S$ be the rectifiable subset carrying the defect measure $\nu=\Theta \mathcal{H}^{n-4}\lfloor S$ of the sequence $A_{i}$ of Yang-Mills connections. For $\mathcal{H}^{n-4}$ a.e. $x_{0} \in S, S$ admits a unique tangent plane $P_{0}$ at $x_{0}$, moreover for $\mathcal{H}^{n-4}$ a.e. $x_{0} \in S$ we 
have

$$
\lim _{r \rightarrow 0} \frac{1}{r^{n-4}} \int_{B_{r}\left(x_{0}\right)}\left|F_{A}\right|^{2}=0
$$

where $A$ is the limiting admissible connection. This last fact is a consequence of a Federer-Zimmer type covering argument detailed in [19] p. 222. For any $\Lambda>0$ we denote by $G_{\Lambda}$ the following subset $B^{n}$ which has to be a subset of $S$

$$
G_{\Lambda}=\left\{x ; \quad \liminf _{r \rightarrow 0} \liminf _{i \rightarrow+\infty} \frac{1}{r^{n-4}} \int_{B_{r}(x)}\left|\nabla_{A_{i}} \nabla_{A_{i}} F_{A_{i}}\right| \geq \Lambda\right\}
$$

For any $\delta>0$ we may assign to each $x$ in $G_{\Lambda}$ a positive radius $r_{x}<\delta$ such that

$$
\forall r<r_{x} \quad \liminf _{i \rightarrow+\infty} \frac{1}{r^{n-4}} \int_{B_{r}(x)}\left|\nabla_{A_{i}} \nabla_{A_{i}} F_{A_{i}}\right| \geq \frac{\Lambda}{2}
$$

From $\left(B_{r_{x}}(x)\right)_{x \in G_{\Lambda}}$ we may extract a Besicovitch covering of $G_{\Lambda}$, $\left(B_{r_{k}}\left(x_{k}\right)\right)_{k \in K}$, where $K$ is at most countable, such that each point of $B^{n}$ is covered by a number of balls $B_{r_{k}}\left(x_{k}\right)$ bounded by a universal number. Then, for any finite subfamilly $J$ of $K$, we have an $n_{J}$ such that

$$
\forall j \in J \quad \forall i \geq n_{J} \quad \int_{B_{r_{j}}\left(x_{j}\right)}\left|\nabla_{A_{i}} \nabla_{A_{i}} F_{A_{i}}\right| \geq \frac{r_{j}^{n-4} \Lambda}{4}
$$

summing over $j$ because of the Besicovitch property we deduce that

$$
\sum_{j \in J} r_{j}^{n-4} \leq C \frac{\left\|\nabla_{A_{i}} \nabla_{A_{i}} F_{A_{i}}\right\|_{L^{1}}\left(B^{n}\right)}{\Lambda}
$$

where $C$ is a universal number independent on $J$. Thus we deduce $\sum_{k \in K} r_{k}^{n-4} \leq \frac{C}{\Lambda}$ and since $\delta$ can be chosen as small as we want, we have $\mathcal{H}^{n-4}\left(G_{\Lambda}\right) \leq C / \Lambda$. From this we deduce

$$
\mathcal{H}^{n-4}\left(\cap_{\Lambda} G_{\Lambda}\right)=0
$$

In other words we have For $\mathcal{H}^{n-4}$ a.e. $x_{0} \in \Sigma$

$$
\liminf _{r \rightarrow 0} \liminf _{i \rightarrow+\infty} \frac{1}{r^{n-4}} \int_{B_{r}\left(x_{0}\right)}\left|\nabla_{A_{i}} \nabla_{A_{i}} F_{A_{i}}\right|<+\infty .
$$


Choose now $x_{0}$ in $\Sigma$ such that $\Sigma$ admits a tangent plane $P_{0}$ at $x_{0}$ and both (II.1) and (II.2) hold. Let $\phi_{r}(x)=r x+x_{0}$, the previous statements imply

$$
\begin{aligned}
& \lim _{r \rightarrow 0} \lim _{i \rightarrow+\infty} \frac{1}{r^{n-4}} \phi_{r}^{*} \mu_{i}=\lim _{r \rightarrow 0} \frac{1}{r^{n-4}} \phi_{r}^{*}\left|F_{A}\right|^{2} d x+\frac{1}{r^{n-4}} \phi_{r}^{*} \Theta \mathcal{H}^{n-4}\lfloor S \\
& =\Theta\left(x_{0}\right) \mathcal{H}^{n-4}\left\lfloor P_{0}\right.
\end{aligned}
$$

where $\mu_{i}$ denotes the measure $\mu_{i}=\left|F_{A_{i}}\right|^{2} d x$ ( $d x$ is the Lebesgue measure). From (II.2) there exists $r_{k} \rightarrow 0$ as $k \rightarrow+\infty$ such that for all $k$

$$
\liminf _{i \rightarrow+\infty} \frac{1}{r_{k}^{n-4}} \int_{B_{r_{k}}\left(x_{0}\right)}\left|\nabla_{A_{i}} \nabla_{A_{i}} F_{A_{i}}\right| \leq C
$$

where $C$ is fixed. Then for any $k$ we may find $i_{k}$ large enough such that

$$
\lim _{k \rightarrow+\infty} \frac{1}{r_{k}^{n-4}} \phi_{r_{k}}^{*} \mu_{i_{k}}=\Theta\left(x_{0}\right) \mathcal{H}^{n-4}\left\lfloor P_{0}\right.
$$

and

$$
\forall k \quad \frac{1}{r_{k}^{n-4}} \int_{B_{r_{k}}\left(x_{0}\right)}\left|\nabla_{A_{i_{k}}} \nabla_{A_{i_{k}}} F_{A_{i_{k}}}\right| \leq 2 C .
$$

Let $\tilde{A}_{k}(x)=r_{k} A_{i_{k}}\left(r_{k} x+x_{0}\right)$ for $x$ in $B_{1}^{n}(0)$, we deduce that

$$
\left|F_{\tilde{A}_{k}}\right|^{2}(x) d x=\frac{1}{r_{k}^{n-4}} \phi_{r_{k}}^{*} \mu_{i_{k}} \longrightarrow \Theta\left(x_{0}\right) \mathcal{H}^{n-4}\left\lfloor P_{0}\right.
$$

and

$$
\int_{B_{1}^{n}}\left|\nabla_{\tilde{A}_{k}} \nabla_{\tilde{A}_{k}} F_{\tilde{A}_{k}}\right| \leq 2 C .
$$

Thus modulo an adapted rescaling of $A_{i}$ at such $x_{0}$, our tangent defect measure $\Theta^{n-4}\left(\mu, x_{0}\right)=\Theta\left(x_{0}\right) \mathcal{H}^{n-4}\left\lfloor P_{0}\right.$ is the defect measure of a sequence of Yang-Mills connections converging weakly to a flat connection $A\left(F_{A}=0\right)$ :

$$
\left|F_{A_{i}}\right|^{2} d x \rightarrow \Theta\left(x_{0}\right) \mathcal{H}^{n-4}\left\lfloor P_{0} \quad \text { in } B_{1} .\right.
$$

To simplify notations we can take $x_{0}=0$ and $P_{0}$ to be the $n$-4-dimensional plane $\left\{x_{i}=0\right\}$ for $i=n-3 \cdots n$. We will simply denote $\Theta\left(x_{0}\right)$ by $\Theta$ that we assume to be non zero. Observe that we can also have chosen $x_{0}$ 
and the rescaled rate such that our rescaled connection $A_{i}$ verifies for any $k=1 \cdots n-4$

$$
\lim _{i \rightarrow+\infty} \int_{B_{1}} \mid \frac{\partial}{\partial x_{k}}\left\lfloor\left. F_{A_{i}}\right|^{2} d x=0\right.
$$

(cf. Lemma 3.3.2 of [19]) where $\frac{\partial}{\partial x_{k}}\left\lfloor F_{A_{i}}\right.$ denotes the contraction of $\frac{\partial}{\partial x_{k}}$ and $F_{A_{i}}$ that lies in $\Omega^{1}\left(\mathfrak{G}_{E}\right)$.

We use the following notations $X_{1}=\left(x_{1} \cdots x_{n-4}\right)$ and $X_{2}=$ $\left(x_{n-3} \cdots x_{n}\right)$. Let

$$
f_{i}\left(X_{1}\right)=\int_{\left\{X_{1}\right\} \times B_{1}^{4}(0)} \sum_{k=1}^{n-4} \mid \frac{\partial}{\partial x_{k}}\left\lfloor\left. F_{A_{i}}\right|^{2} .\right.
$$

Since $f_{i} \rightarrow 0$ in $L^{1}\left(B_{1}^{n-4}(0)\right)$, its maximal function

$$
M f_{i}\left(X_{1}\right)=\sup _{r>0} \frac{1}{r^{n-4}} \int_{B_{r}^{n-4}\left(X_{1}\right)} f_{i}
$$

tends to zero in $L_{\text {weak }}^{1}$ :

$$
\lim _{i \rightarrow+\infty}\left\|\lambda\left|\left\{X_{1} ; M f_{i}\left(X_{1}\right) \geq \lambda\right\}\right|\right\|_{L^{\infty}(\mathbb{R})}=0 .
$$

Thus there exists a set $E_{i},\left|E_{i}\right| \geq 0,99\left|B_{1}^{n-4}(0)\right|$ such that

$$
\left\|M f_{i}\right\|_{L^{\infty}\left(E_{i}\right)} \rightarrow 0 .
$$

Using the fact that $\left\|\nabla_{A_{i}} \nabla_{A_{i}} F_{A_{i}}\right\|_{L^{1}\left(B_{1}^{n}\right)}$ is uniformly bounded and Kato inequality $|d| \nabla_{A_{i}} F_{i}|| \leq\left|\nabla_{A_{i}} \nabla_{A_{i}} F_{i}\right|$, (we keep denoting the curvature of $A_{i}$ by $F_{i}$ ) we deduce that

$$
\int_{B_{1}(0)}\left|\nabla_{A_{i}} F_{i}\right|^{\frac{4}{3}}+|d| \nabla_{A_{i}} F_{i}|| \leq C
$$

where $C$ is independant on $i$. By Fubini there exists a measurable subset $G_{i}$ of $B_{1}^{n-4}(0)$, verifying $\left|G_{i}\right| \geq 0,99\left|B_{1}^{n-4}\right|$ and such that

$$
\forall X_{1} \in G_{i} \quad \int_{\left\{X_{1}\right\} \times B_{1}^{4}(0)}\left|\nabla_{A_{i}} F_{i}\right|^{\frac{4}{3}}+|d| \nabla_{A_{i}} F_{i}|| \leq C
$$

where $C$ is independant on $i$ and $X_{1}$ in $G_{i}$. Using now the embedding of $W^{1,1}\left(B^{4}\right)$ into the Lorentz Space $L^{\frac{4}{3}, 1}\left(B^{4}\right)$ (this embedding was first noticed 
by J. Peetre [15], see also [18] and a short proof in [8] Theorem 3.3.9) we get that

$$
\forall X_{1} \in G_{i} \quad\left\|\nabla_{A_{i}} F_{i}\right\|_{L^{\frac{4}{3}, 1}\left(\left\{X_{1}\right\} \times B_{1}^{4}(0)\right)} \leq C
$$

which yields, by Kato inequality,

$$
\forall X_{1} \in G_{i} \quad\left\|d\left|F_{i}\right|\right\|_{L^{\frac{4}{3}, 1}\left(\left\{X_{1}\right\} \times B_{1}^{4}(0)\right)} \leq C .
$$

$C$ again is independant of $i$. We may also have chosen $G_{i}$ such that, for any $X_{1}$ in $G_{i}, \int_{\left\{X_{1}\right\} \times B_{1}^{4}(0)}\left|F_{i}\right|^{2}$ is bounded independantly of $i$ preserving the size of $G_{i}:\left|G_{i}\right| \geq 0,99\left|B_{1}^{n-4}\right|$. Using now theorem 8 of [18] we get that the $L^{2,1}$ norm of $F_{i}$ on $\left\{X_{1}\right\} \times B_{1}^{4}(0)$ is uniformly bounded

$$
\forall X_{1} \in G_{i} \quad\left\|F_{i}\right\|_{L^{2,1}\left(\left\{X_{1}\right\} \times B_{1}^{4}(0)\right)} \leq C .
$$

We will call a "good slice" a point $X_{1} \in G_{i} \cap E_{i}$ (this set has a measure bounded from bellow independantly of $i$ ).

We claim that for any $X_{1} \in B_{1}^{n-4}(0)$ and any fixed $\delta$, for $i$ large enough,

$$
\sup _{X_{2} \in B_{1}^{4}(0)} \delta^{4-n} \int_{B_{\delta}\left(X_{1}, X_{2}\right)}\left|F_{A_{i}}\right|^{2} \geq \varepsilon(n)
$$

where $\varepsilon(n)$ is the Uhlenbeck constant given in Theorem 2.2.1 of [19]. That yields to the control of the $L^{\infty}$ norm of the curvature in the ball of half radius. If (II.11) would not hold we would contradicts the concentration phenomenon and the fact that $\Theta \neq 0$.

Pick now an arbitrary sequence of "good slices" $\left\{X_{1}^{i}\right\}$ as $i \rightarrow+\infty$. Because of (II.11), since each $A_{i}$ is smooth, we may find a sequence $\delta_{i} \rightarrow 0$ such that

$$
\max _{X_{2}} \delta_{i}^{4-n} \int_{B_{\delta_{i}}\left(X_{1}^{i}, X_{2}\right)}\left|F_{A_{i}}\right|^{2}=\delta_{i}^{4-n} \int_{B_{\delta_{i}}\left(X_{1}^{i}, X_{2}^{i}\right)}\left|F_{A_{i}}\right|^{2}=\frac{\varepsilon(n)}{2}
$$

( $1 / 2$ can be replaced by any positive number between 0 and 1$)$. In other words $X_{2}^{i}$ is located at a bubble of characteristic size $\delta_{i}$. More precisely, if one introduces $B_{i}=\tau_{i}^{*} A_{i}$ where $\tau_{i}: X \rightarrow \delta_{i} X+\left(X_{1}^{i}, X_{2}^{i}\right)$, we have, following [19] p. 237 that, modulo extraction of a subsequence and eventually changes of gauge,

$$
F_{B_{i}} \rightarrow F_{B} \quad \text { in } C_{\mathrm{loc}}^{k}\left(B_{1}^{n-4}(0) \times \mathbb{R}^{4}\right)
$$


moreover $B$ is gauge equivalent to the pull-back by the orthogonal projection map of a non flat Yang-Mills connection on $\{0\} \times \mathbb{R}^{4}$. This is the first bubble we detect.

At this stage of the proof it is tempted to compare $\Theta$ and $\int_{\{0\} \times \mathbb{R}^{4}}\left|F_{B}\right|^{2}$ and to deduce from the above convergence that $\Theta \geq \int_{\{0\} \times \mathbb{R}^{4}}\left|F_{B}\right|^{2}$. This will be a consequence of the following lemma

Lemma II.1. Let $A_{i}$ be a sequence of Yang-Mills connections on $B_{1}^{n}$ such that

$$
\left|F_{A_{i}}\right|^{2} d x \longrightarrow \Theta_{0} \mathcal{H}^{n-4}\left\lfloor P_{0}\right.
$$

as Radon measures, where $\Theta_{0}$ is a positive constant and $P_{0}$ is the $n-4$ plane $\left\{x_{k}=0 ; k=n-3 \cdots n\right\}$, assume also that

$$
\forall k=1 \cdots n-4 \quad \frac{\partial}{\partial x_{k}}\left\lfloor F_{A_{i}} \longrightarrow 0 \quad \text { in } L^{2}\left(B_{1}^{n}\right)\right.
$$

then for a.e. $X_{1} \in P_{0}$

$$
\lim _{i \rightarrow+\infty} \int_{\left\{X_{1}\right\} \times B_{1}^{4}(0)}\left|F_{A_{i}}\right|^{2}=\Theta_{0} .
$$

Proof of Lemma II.1. The idea of the proof comes from Lin's work [9] in the context of stationary harmonic maps. Computations which are required to prove the lemma can be found in [19] part 3 .

Let $\phi \in C_{0}^{\infty}\left(B_{1}^{4}(0)\right)$ such that $\phi\left(X_{2}\right) \equiv 1$ in $B_{1 / 2}^{4}(0)$, introduce

$$
m_{i}\left(X_{1}\right)=\int_{B_{1}^{4}(0)} \phi^{2}\left|F_{A_{i}}\right|^{2}\left(X_{1}, X_{2}\right) d X_{2}
$$

following (3.3.19) of [19] we obtain that $m_{i}$ solves the following equation:

$$
\operatorname{grad} m_{i}=f_{i}+\operatorname{div} u_{i}
$$

where $f_{i}: B_{1}^{n-4} \rightarrow \mathbb{R}^{n-4}$ and $u_{i}: B_{1}^{n-4} \rightarrow \mathbb{R}^{n-4} \times \mathbb{R}^{n-4}$ verify

$$
\left\|f_{i}\right\|_{L^{1}\left(B_{1}^{n-4}\right)}+\left\|u_{i}\right\|_{L^{1}\left(B_{1}^{n-4}\right)} \longrightarrow 0 .
$$

(II.15) is of course used to establish (II.18.) From Allard strong constancy Lemma [1] we deduce the existence of a constant $C_{i}$ such that

$$
\left\|m_{i}-C_{i}\right\|_{L_{\mathrm{loc}}^{1}\left(B_{1}^{n-4}\right)} \longrightarrow 0 .
$$


Since $\int_{B_{r}^{n-4}} m_{i} \rightarrow\left|B_{r}^{n-4}\right| \Theta_{0}$, we deduce that $C_{i} \rightarrow \Theta_{0}$ as $i \rightarrow+\infty$ and Lemma II.3 is proved since $\left|F_{A_{i}}\right|$ converges to 0 in $B_{1}^{n} \backslash P_{0}$.

Because of (II.16) we may of course choose $X_{1}^{i}$ in $E_{i} \cap G_{i}$ such that

$$
\lim _{i \rightarrow \infty} \int_{\left\{X_{1}^{i}\right\} \times B_{1}^{4}(0)}\left|F_{A_{i}}\right|^{2}=\Theta .
$$

Thus at this stage of the proof we have shown that

$$
\Theta \geq \int_{\mathbb{R}^{4}}\left|F_{B}\right|^{2}
$$

where $B$ is a non flat YM-connection of $\mathbb{R}^{4}$.

We can restate the goal to achieve in order to prove Theorem I.2: the aim is to show that

$$
\lim _{i \rightarrow+\infty} \int_{\left\{X_{1}^{i}\right\} \times B_{1}^{4}(0)}\left|F_{A_{i}}\right|^{2}=\sum_{j=1}^{m} \int_{\mathbb{R}^{4}}\left|F_{B_{j}}\right|^{2}
$$

where $F_{B_{j}}$ are non flat Yang-Mills connections on $\mathbb{R}^{4}$. This will be shown by induction on the total number of bubbles $m$. Indeed we know that each concentration generating a bubble has a cost of energy bounded from bellow by a positive number $\varepsilon(4)$ (recall that $\varepsilon(4)$ is the Uhlenbeck constant in 4 dimension see [20]). Thus the number of possible bubbles is uniformly bounded.

Assume first we have only one bubble which is the one already detected of characteristic size $\delta_{i}$. Clearly we have

$$
\int_{\mathbb{R}^{4}}\left|F_{B}\right|^{2}=\lim _{R \rightarrow+\infty} \lim _{i \rightarrow+\infty} \int_{\left\{X_{1}^{i}\right\} \times B_{R \delta_{i}}^{4}\left(X_{2}^{i}\right)}\left|F_{A_{i}}\right|^{2} .
$$

In order to prove that

$$
\Theta=\lim _{i \rightarrow+\infty} \int_{\left\{X_{1}^{i}\right\} \times B_{1}^{4}(0)}\left|F_{A_{i}}\right|^{2}=\int_{\mathbb{R}^{4}}\left|F_{B}\right|^{2}
$$

we have to prove that

$$
\lim _{R \rightarrow+\infty} \lim _{i \rightarrow+\infty} \int_{\left\{X_{1}^{i}\right\} \times B_{1}^{4} \backslash B_{R \delta_{i}}^{4}\left(X_{2}^{i}\right)}\left|F_{A_{i}}\right|^{2}=0
$$


in other words that there is no "neck" formation and no loss of energy which is not quantized (see [7] and [16] for "neck" phenomenon in bubble trees).

To simplify notations we take $\left(X_{1}^{i}, X_{2}^{i}\right)$ to be the origin $(0,0)$.

We claim that for any $\varepsilon$ there are sufficiently large $R>0$ and $i_{0} \in \mathbb{N}$ such that

$$
\begin{aligned}
\forall i \geq & i_{0} \quad \forall R \delta_{i} \leq r \leq \frac{1}{2} \\
& \frac{1}{r^{n-4}} \int_{B_{r}^{n-4}(0)} \int_{B_{2 r}^{4}(0) \backslash B_{r}^{4}(0)}\left|F_{A_{i}}\right|^{2} \leq \varepsilon .
\end{aligned}
$$

Assume this is not the case, then we may find an $\varepsilon_{0}>0$, a subsequence $i^{\prime} \rightarrow+\infty$ (still denoted $i$ ) and a sequence $r_{i}$ such that

$$
\begin{aligned}
& \frac{1}{r_{i}^{n-4}} \int_{B_{r_{i}}^{n-4}(0)} \int_{B_{2 r_{i}}^{4}(0) \backslash B_{r_{i}}^{4}(0)}\left|F_{A_{i}}\right|^{2} \geq \varepsilon_{0} \\
& \frac{r_{i}}{\delta_{i}} \rightarrow+\infty .
\end{aligned}
$$

(We may allways assume that $r_{i} \rightarrow 0$ since $F_{A_{i}} \rightarrow 0$ in $B^{n} \backslash P_{0}$.) Let $\lambda_{i} \rightarrow 0$ such that $\frac{r_{i}}{\lambda_{i}}=o(1)$ and $\lambda_{i} \frac{r_{i}}{\delta_{i}} \rightarrow+\infty$. Consider the dilation map $\tau_{i}(X)=r_{i} X$ in $\mathbb{R}^{n}$ and let $B_{i}=\tau_{i}^{*} A_{i}$, thus $B_{i}$ is a Yang-Mills connection on $B_{1}^{n-4}(0) \times B_{2 / \lambda_{i}}^{4}(0) \backslash B_{\lambda_{i}}^{4}$ and satisfies

$$
\int_{B_{1}^{n-4}(0)} \int_{B_{2}^{4}(0) \backslash B_{1}^{4}(0)}\left|F_{B_{i}}\right|^{2} \geq \varepsilon_{0} .
$$

Taking into account the translation by $\left(X_{1}^{i}, X_{2}^{i}\right)$, (II.5) implies

$$
\lim _{i \rightarrow+\infty} \sup _{r} \frac{1}{r^{n-4}} \int_{B_{r}^{n-4}} \int_{B_{2 / \lambda_{i}}^{4}(0) \backslash B_{\lambda_{i}}^{4}(0)} \mid \frac{\partial}{\partial x_{k}}\left\lfloor\left. F_{B_{i}}\right|^{2}=0 .\right.
$$

Thus, from Proposition 3.1.2 of [19], modulo extraction of a subsequence and eventually changes of gauge, we have

$$
F_{B_{i}} \rightarrow F_{B_{\infty}} \quad \text { in } L_{\text {loc }}^{2}\left(B_{1}^{n-4}(0) \times \mathbb{R}^{4} \backslash\{0\}\right)
$$

where

$$
\begin{aligned}
& \forall k=1 \cdots n-4 \quad \frac{\partial}{\partial x_{k}}\left\lfloor F_{B_{\infty}}=0\right. \\
& \int_{B_{1}^{n-4}(0) \times \mathbb{R}^{4}}\left|F_{B_{\infty}}\right|^{2}<+\infty \\
& B_{\infty} \text { is an admissible YM connection }
\end{aligned}
$$


arguing like in [19] p. 237 we deduce that $F_{B_{\infty}}$ is gauge equivalent to the pullback of the projection on $\{0\} \times \mathbb{R}^{4}$ of a Yang-Mills connection on $\{0\} \times \mathbb{R}^{4} \backslash\{0\}$ which has to be smooth at $\{0\}$ because of the removability singularity result of Uhlenbeck [20]. Thus $B_{\infty}$ is gauge equivalent to the pull-back of an $\mathbb{R}^{4}$ YM-connection. If $B_{\infty}$ is not flat we already get a contradiction because we just exhibited a new bubble. If $F_{B_{\infty}}$ is flat, that means that the convergence of $F_{B_{i}}$ to $F_{B_{\infty}}$ admits a non zero defect measure

$$
\left|F_{B_{i}}\right|^{2} \rightarrow \nu=f(x) \mathcal{H}^{n-4}\lfloor\Sigma
$$

and because of (II.29) we easily deduce, using arguments like in the proof of Lemma II.1, that $\Sigma$ is contained in $n-4$-planes $P_{i}$ parallel to $\left\{x_{k}=0 ; k \geq\right.$ $n-3\}$ and that $f$ is constant on each of the $P_{i}$ s:

$$
\nu=\sum_{j} C_{j} \mathcal{H}^{n-4}\left\lfloor P_{j}\right.
$$

where the $C_{j}$ are non zero constants. Following again [19] page 235 we get a new bubble and this contradict our assumption that there is only one bubble.

Thus in any cases (II.27) yields a contradiction and (II.26) holds. For $\varepsilon$ small enough in (II.26) we are in the setting of Uhlenbeck $\varepsilon$-regularity theorem ([19] Theorem 2.2.1 or[14]) and we deduce the existence of $\varepsilon_{0}$ such that for any $\varepsilon<\varepsilon_{0}$, there exists $R>0$ and $i_{0} \in \mathbb{N}$ such that

$$
\forall i \geq i_{0} \quad \forall|X| \geq R \delta_{i} \quad|X|^{4}\left|F_{A_{i}}\right|^{2} \leq \varepsilon
$$

restricting $F_{A_{i}}$ to the plane $\{0\} \times \mathbb{R}^{4}$ we deduce from (II.32)

$$
\left\|F_{A_{i}}\right\|_{L^{2, \infty}\left(\{0\} \times B_{1 / 2}^{4} \backslash B_{R \delta_{i}}^{4}\right)}^{2} \leq \varepsilon
$$

where $L^{2, \infty}$ is the Lorentz Space defined for instance in [17]. Combining (II.33) with (II.10) and using the $L^{2, \infty}-L^{2,1}$ duality (see [17]) we deduce that for every $\varepsilon<\varepsilon_{0}$ there exists an $R$ such that for any $i \geq i_{0}$ we have

$$
\left\|F_{A_{i}}\right\|_{L^{2}\left(B_{1 / 2}^{4} \backslash B_{R \delta_{i}}^{4}\right)}^{2} \leq C \varepsilon .
$$

This implies (II.25) and Theorem I.2 is proved in the case of 1 bubble.

The case of more than 1 bubble can be handeled in a very similar way and we just give few details for $m=2$.

The proof starts the same untill (II.26) which cannot hold anymore otherwise we would have had 1 bubble only as it is explained above. Thus we 
may extract $r_{i}$ such that (II.27) holds. We have two possibilities the first: $F_{B_{i}}$ has no defect measure and the convergence is strong and it remains to show that

$$
\lim _{R \rightarrow+\infty} \lim _{i \rightarrow+\infty} \int_{\{0\} \times B_{r_{i} / R}^{4} \backslash B_{R \delta_{i}}^{4}}\left|F_{B_{i}}\right|^{2}=0
$$

and

$$
\lim _{R \rightarrow+\infty} \lim _{i \rightarrow+\infty} \int_{\{0\} \times B_{1 / 2}^{4} \backslash B_{R r_{i}}^{4}}\left|F_{B_{i}}\right|^{2}=0 .
$$

This can be done exactly like in the proof of (II.27) since we already got the second bubble given by $F_{B_{\infty}}$. The second situation is when $\left|F_{B_{i}}\right|^{2}$ has some non-zero defect measure and it is given by (II.31). We need to have $F_{B_{\infty}}=0$ otherwise there would be too many bubbles. Thus since $F_{B_{\infty}}=0$ we are back to the case $m=1$. In this case also one has to establish (II.35) and (II.36) and this can be done again following word by word the proof of (II.27). The case $m=2$ is proved. For larger $m$ one follows exactly the same strategy.

\section{The conformal dimension $n=4$.}

In this part we explain, in the case $n=4$ how we can omitt the hypothesis on the $W^{2,1}$ bound of the curvature to prove Theorem I.2. Theorem I.2 was not known at the conformal dimension $n=4$ in general but only established for ASD connections in this dimension (see the analysis in [20], [21] and [6]). We follow the same strategy as the one developped in part II and we keep denoting $\delta_{i}$ the caracteristic size of the first bubble given by (II.12) that we assume to be centered at the origin. In order to clarify the presentation we assume that there is only one bubble so that (II.26) holds:

$$
\forall \varepsilon \quad \exists R \equiv R_{\varepsilon} \quad \text { s.t. } \quad \forall i \geq i_{0} \quad \forall r \text { s.t. } R_{\varepsilon} \delta_{i} \leq r \leq 4
$$

$$
\int_{B_{16 r}^{4}(0) \backslash B_{r}^{4}(0)}\left|F_{A_{i}}\right|^{2} \leq \varepsilon .
$$

We claim the following lemma holds:

Lemma III.1. There exists $\varepsilon_{0}>0$ such that for any $\varepsilon<\varepsilon_{0}$ There exists a trivialization of the bundle on $B^{4} \backslash B_{R_{\varepsilon} \delta_{i}}(0)$ such that, in this trivialization

$$
\left\|A_{i}\right\|_{W^{1,2}\left(B^{4} \backslash B_{R_{\varepsilon} \delta_{i}}(0)\right)}
$$


is uniformly bounded, independently on $i$ and $\varepsilon$.

Proof of Lemma III.1. We first have a fixed trivialisation over $B^{4}$ (coming from the bundle we fixed) in which we have no control of the sequence of connections $A_{i}$ which are 1-forms defined on all of $B^{4}$ into the Lie algebra $\mathfrak{G}$ of the structure group $G$. Let $R$ given by (III.1) for an $\varepsilon$ that will be fixed later on in the proof. We introduce the following family of annulii centered at $0: T^{k}=B_{2^{-k+2}}(0) \backslash B_{2^{-k-2}}(0)$ for the integers going from 0 to $N_{i}=\left[\log _{2}\left(1 / R \delta_{i}\right)+2\right]([s]$ denotes the integer part of the real $s)$. Applying Uhlenbeck's Theorem 1.3 in [21], for $\varepsilon$ chosen small enough, independent on $i$ or $k$, for any $k=0 \cdots N_{i}$, there exists a change of gauge $\sigma_{i}^{k}$ on $T^{k}$ such that if we denote $A_{i}^{k}$ the connection expressed in this gauge $\left(A_{i}^{k}=\right.$ $\left.\left(\sigma_{i}^{k}\right)^{-1} A_{i} \sigma_{i}^{k}+\left(\sigma_{i}^{k}\right)^{-1} d \sigma_{i}^{k}\right)$ we have

$$
\int_{T_{i}^{k}}\left|\nabla A_{i}^{k}\right|^{2}+\frac{\left|A_{i}^{k}\right|^{2}}{|x|^{2}} \leq c \int_{T_{i}^{k}}\left|F_{A_{i}}\right|^{2} \leq c \varepsilon
$$

(observe that we have no control on the $\sigma_{i}^{k}$ but only on the transition functions $\left(\sigma_{i}^{k}\right)^{-1} \sigma_{i}^{k-1}$ as we will see bellow). Using again Uhlenbeck regularity theorem in ([20] p. 18), we have the estimate in the smaller annuli $\hat{T}_{i}^{k}=B_{2^{-k+1}}(0) \backslash B_{2^{-k-1}}(0)$

$$
\left|\nabla^{l} A_{i}^{k}\right| \leq C_{l} \frac{\varepsilon^{1 / 2}}{|x|^{l+1}}
$$

On $\hat{T}^{k} \cap \hat{T}^{k-1}=B_{2^{-k+1}}(0) \backslash B_{2^{-k}}(0)$ let $g_{i}^{k, k-1}$ be the transition function given by

$$
g_{i}^{k, k-1}=\left(\sigma_{i}^{k}\right)^{-1} \sigma_{i}^{k-1}
$$

We have

$$
d g_{i}^{k, k-1}=g_{i}^{k, k-1} A_{i}^{k-1}-A_{i}^{k} g_{i}^{k, k-1}
$$

and from (III.3) we deduce

$$
\left|\nabla g_{i}^{k, k-1}\right| \leq C \varepsilon /|x| \quad \text { and } \quad\left|\nabla^{2} g_{i}^{k, k-1}\right| \leq C \varepsilon /|x|^{2} .
$$

Then there exists $\bar{g}_{i}^{k, k-1}$ in $G$ such that

$$
\left\|g_{i}^{k, k-1}-\bar{g}_{i}^{k, k-1}\right\|_{L^{\infty}\left(\hat{T}^{k} \cap \hat{T}^{k-1}\right)} \leq C \varepsilon .
$$


Let $h^{k}=\prod_{l \leq k-1} \bar{g}_{i}^{l, l-1}$ (ordered product $\bar{g}_{i}^{k-1, k-2} \bar{g}_{i}^{k-2, k-3} \ldots$...), replacing $g_{i}^{k, k-1}$ by $\tilde{g}_{i}^{k, k-1}=\left(h^{k}\right)^{-1} g_{i}^{k, k-1} h^{k-1}$ we have

$$
\left\|\tilde{g}_{i}^{k, k-1}-\mathrm{id}\right\|_{L^{\infty}\left(\hat{T}^{k} \cap \hat{T}^{k-1}\right)}=\left\|\bar{g}_{i}^{k, k-1} g_{i}^{k, k-1}-\mathrm{id}\right\|_{L^{\infty}\left(\hat{T}^{k} \cap \hat{T}^{k-1}\right)} \leq \varepsilon .
$$

So changing $\sigma_{i}^{k}$ into $\sigma_{i}^{k} h^{k}$, we may assume $\bar{g}_{i}^{k, k-1}=\mathrm{id}$. We assume now that $\varepsilon$ is chosen small enough so that Uhlenbeck's Theorem (III.2) applies and so that, in view of (III.6), $g_{i}^{k, k-1}$ is in the neighborhood of the identity where exp is invertible. The goal is to express $g_{i}^{k, k-1}$ as transition functions (eventualy on smaller intersecting set than the $\hat{T}^{k} \cap \hat{T}^{k-1}$ ) of a sequence of trivialisations $\rho_{i}^{k}$ on which we have some control. Let $\phi$ be a smooth map on $B_{2}(0)$ such that $\phi \equiv 1$ on $B_{2} \backslash B_{2^{\frac{1}{2}}}$ and $\phi \equiv 0$ on $B_{1}$. Denote by $\phi^{k}(x)=\phi\left(2^{k} x\right) . \rho^{k}$ is now defined by induction on $k$. Let $\rho_{i}^{0} \equiv 1$. assume now $\rho_{i}^{k-1}$ is constructed, we denote

$$
Y_{i}^{k}=\exp ^{-1}\left(\rho_{i}^{k-1} g_{i}^{k, k-1}\right)
$$

and we choose on $\tilde{T}^{k}=B_{2^{-k+1}}(0) \backslash B_{2^{-k-1 / 2}}(0), \rho^{k}:=\exp \left(-\phi^{k} Y_{i}^{k}\right)$. Since $\rho^{k-1} \equiv \mathrm{id}$ on $B_{2^{-k+1}}$ we have

$$
\left|\nabla^{l} \rho_{i}^{k}\right| \leq C \sum_{j=0}^{l}\left|\nabla^{j} \phi^{k}\right|\left|\nabla^{l-j} Y^{k}\right| \leq C \sum_{j=0}^{l} 2^{j k}\left|\nabla^{l-j} g_{i}^{k, k-1}\right| .
$$

Thus from (III.6) and (III.7), we have for $l=0,1,2$

$$
\left|\nabla^{l} \rho_{i}^{k}\right| \leq \varepsilon /|x|^{l}
$$

and from (III.2) and (III.4)

$$
\int_{\tilde{T}^{k}}\left|\nabla^{2} \rho_{i}^{k}\right|^{2}+\frac{\left|\nabla \rho_{i}^{k}\right|^{2}}{|x|^{2}} \leq C \int_{T^{k}}\left|F_{A_{i}}\right|^{2}
$$

Moreover on $\tilde{T}^{k} \cap \tilde{T}^{k-1}=B_{2^{-k+1}} \backslash B_{2^{-k+1 / 2}}$, by construction $g_{i}^{k, k-1}=$ $\left(\rho_{i}^{k}\right)^{-1} \rho_{i}^{k-1}$. Let $\tilde{F}_{i}$ be the two form on $B_{2} \backslash B_{R \delta_{i}}$ into the Lie algebra of $G$ that coincide with $\left(\rho_{i}^{k-1}\right)^{-1} F_{i}^{k-1} \rho_{i}^{k-1}=\left(\rho_{i}^{k}\right)^{-1} F_{i}^{k} \rho_{i}^{k}$ in $t i T^{k}$, it is our original sequence of curvatures $F_{A_{i}}$ expressed in the trivialisation that coincide with $\rho_{i}^{k} \sigma_{i}^{k}$ on the $t i T^{k}$ 's. the corresponding 1-form expressing the connection in this gauge is $\tilde{A}_{i}=\left(\rho_{i}^{k}\right)^{-1} A_{i}^{k} \rho_{i}^{k}+\left(\rho_{i}^{k}\right)^{-1} d \rho_{i}^{k}$ on $\tilde{T}^{k}$. Combining 
(III.2) and (III.8) and (III.9), we have

$$
\begin{aligned}
& \int_{B_{2} \backslash B_{2 R \delta_{i}}} \frac{\left|\tilde{A}_{i}\right|^{2}}{|x|^{2}}+\left|\nabla \tilde{A}_{i}\right|^{2} \leq C \sum_{k=0}^{N_{i}} \int_{T^{k}} \frac{\left|A_{i}^{k}\right|^{2}}{|x|^{2}}+\left|\nabla A_{i}^{k}\right|^{2} \\
& \leq C \int_{B_{4} \backslash B_{R \delta_{i}}}\left|F_{A_{i}}\right|^{2} .
\end{aligned}
$$

Finally the $L^{2,1}$ estimate of the curvature and the quantization property (Theorem I.1 for $n=4$ ) is a consequence of inequality (III.10) and the following observation.

Lemma III.2. Let $A$ be the 1-form of a Yang-Mills connection on a given trivialization of a principal bundle over $B^{4}$, the following estimate holds

$$
\left\|F_{A}\right\|_{L^{2,1}\left(B_{1 / 2}^{4}\right)} \leq C\left[\|A\|_{W^{1,2}\left(B^{4}\right)}+\|A\|_{W^{1,2}\left(B^{4}\right)}^{3}\right]
$$

where $F_{A}$ is the curvature $F_{A}=d A+[A, A]$ and \|\|$_{L^{2,1}}$ denotes the Lorentz norm of the space dual to the weak $L^{2}$ space.

Proof of Lemma III.2. Using whitney extension theorem we may find an extension $\tilde{A}$ of $A$ on all of $\mathbb{R}^{4}$ such that $\|\tilde{A}\|_{W^{1,2}\left(\mathbb{R}^{4}\right)} \leq\|A\|_{W^{1,2}\left(B^{4}\right)}$. Then Using the Lorentz-Sobolev embeding of J. Peetre [15], we have

$$
\|A\|_{L^{4,2}\left(B^{4}\right)} \leq\|\tilde{A}\|_{L^{4,2}\left(\mathbb{R}^{4}\right)} \leq C\|\tilde{A}\|_{W^{1,2}\left(\mathbb{R}^{4}\right)}
$$

where $L^{4,2}$ denotes the Lorentz Space of function $f$ such that $\int_{\mathbb{R}} f_{*}^{2}(t) t^{-1 / 2}<$ $+\infty\left(f_{*}\right.$ is the decreasing rearrengement of $\left.f\right)$. Now using the following property on multiplication of functions in Lorentz Spaces

$$
\forall a, b \text { funct. on } B^{4} \quad\|a b\|_{L^{4 / 3,1}\left(B^{4}\right)} \leq\|a\|_{L^{4,2}\left(B^{4}\right)}\|b\|_{L^{2,2}\left(B^{4}\right)}
$$

$\left(L^{2,2}\right.$ is the usual $L^{2}$ Space) see [18]. Since $F=d A+[A, A]$ solves

$$
d F=-[A, F] \quad \text { and } \quad d^{*} F=-*[A, * F] .
$$

Combining (III.12) and (III.13) we get

$$
\|d F\|_{L^{4 / 3,1}\left(B^{4}\right)}+\left\|d^{*} F\right\|_{L^{4 / 3,1}\left(B^{4}\right)} \leq\|A\|_{W^{1,2}\left(B^{4}\right)}\|F\|_{L^{2}\left(B^{4}\right)} .
$$

let $G$ on $B^{4}$ such that $d G=d F, d^{*} G=d^{*} F$ and $\iota_{\partial B^{4}}^{*} G=0$ (where $\iota_{\partial B^{4}}$ is the embedding of $\partial B^{4}$ in $\left.B^{4}\right)$. The operator that assign to the pair $\left(d G, d^{*} G\right)$ 
the tensor $\nabla G=\sum_{k=1}^{4} \frac{\partial G}{\partial x_{k}} \otimes d x_{k}$ is a weak $(r, r)$ operator for $r$ between 1 and $\infty$, then using Theorem 3.15 Chapter V of [17] we obtain

$$
\begin{aligned}
& \|G\|_{L^{2}\left(B^{4}\right)}+\|\nabla G\|_{L^{4 / 3,1}\left(B^{4}\right)} \leq C\|d G\|_{L^{4 / 3,1}\left(B^{4}\right)}+\left\|d^{*} G\right\|_{L^{4 / 3,1}\left(B^{4}\right)} \\
& \quad \leq\|A\|_{W^{1,2}\left(B^{4}\right)}\|F\|_{L^{2}\left(B^{4}\right)} .
\end{aligned}
$$

Since $F-G$ is an harmonic form on $B^{4}$ with an $L^{2}$ control on $F$ and an $L^{2}$ control on $G$ we get

$$
\|\nabla(F-G)\|_{L^{4 / 3,1}\left(B_{1 / 2}^{4}\right)} \leq C\left(\|A\|_{W^{1,2}\left(B^{4}\right)}+1\right)\|F\|_{L^{2}\left(B^{4}\right)} .
$$

Combining (III.15) and (III.16) we obtain

$$
\|\nabla F\|_{L^{4 / 3,1}\left(B_{1 / 2}^{4}\right)} \leq C\left(\|A\|_{W^{1,2}\left(B^{4}\right)}+1\right)\|F\|_{L^{2}\left(B^{4}\right)} .
$$

Using now the Lorentz-Sobolev embedding of J. Peetre (see [18] Theorem 8 ) we get the desired estimate on the $L^{2,1}$ norm of the curvature and Lemma III.2 is proved.

We show how to adapt the arguments above to the present situation: we have only a gauge in $B_{1}^{4} \backslash B_{R \delta_{i}}^{4}$ in which we control the $W^{1,2}$ norm of the Gauge independently of $i$ and $\varepsilon$. Let $\chi$ be a cut-off function equal to 1 on $B_{1} \backslash B_{2 R \delta_{i}}$ and 0 on $B_{R \delta_{i}}$, such that $\|\nabla \chi\|_{\infty} \leq C / R \delta_{i}$. Let's take $F$ the curvature in the Gauge given by Lemma III.1 and consider $\chi F$. It solves

$$
d(\chi F)=-\chi[A, F]+d \chi \wedge F \quad \text { and } \quad d(*(\chi F))=-\chi[A, * F]+d \chi \wedge * F .
$$

We use the arguments above to deduce that $\|\chi[A, F]\|_{L^{\frac{4}{3}, 1}\left(B^{4}\right)} \leq C$ and similarly $\|\chi[A, * F]\|_{L^{\frac{4}{3}, 1}\left(B^{4}\right)} \leq C$. From the $L^{\infty}$ estimates established for the curvature in the previous part we deduce that

$$
|d \chi \wedge * F| \leq \frac{C}{\left(R \delta_{i}\right)^{3}} \quad \text { and } \quad|d \chi \wedge F| \leq \frac{C}{\left(R \delta_{i}\right)^{3}}
$$

and this terms are supported in the annulus $B_{2 R \delta_{i}} \backslash B_{R \delta_{i}}$ and using the definition of the $L^{\frac{4}{3}, 1}$ norm we easily get that

$$
\|d \chi \wedge * F\|_{L^{\frac{4}{3}, 1}\left(B^{4}\right)} \leq C \quad \text { and } \quad\|d \chi \wedge F\|_{L^{\frac{4}{3}, 1}\left(B^{4}\right)} \leq C
$$


where $C$ is independent on $i$ and $\varepsilon$. So argueing as in the previous lemma we have established that

$$
\|F\|_{L^{2,1}\left(B_{1}^{4} \backslash B_{R \delta_{i}}^{4}\right)} \leq C
$$

where $C$ is independent on $i$ and $\varepsilon$ and the proof of Theorem I.1 follows from the arguments of section 2 .

\section{Some more applications of Lorentz Spaces to the analysis of non-linearities issued from curvatures.}

In this part, which is independent from the rest of the paper, we aim to present how Lorentz spaces are helpful to establish properties relative to strong converging sequences of connections of a given bundle over a riemannian manifold. From now on the Lie group $G$ is a subgoup of $S O(k)$ seen as a submanifold of the vector space of $k$ by $k$ matrices.

We first establish the following result:

Theorem IV.1. Let $Q$ be a principal $G$-bundle over a compact $n$-dimensional riemannian manifold $M$. Assume there exists on $Q$ a sequence of connections $D_{i}$, a fixed covering of $M\left(\mathcal{U}^{\alpha}\right)$ and a trivialization $\sigma_{i}^{\alpha}$ over $\mathcal{U}^{\alpha}$ such that if $A_{i}^{\alpha}=\sigma_{i}^{\alpha} D_{i}\left(\sigma_{i}^{\alpha}\right)^{-1}-d$ is a Coulomb Gauge for $D_{i}\left(d^{*} A_{i}^{\alpha}=0\right)$ that converges strongly to some $A_{\infty}^{\alpha}$ in $W^{1, n / 2}$, then $A_{\infty}^{\alpha}$ defines a connection of the same bundle $Q$ : there exists a connection $D_{\infty}$ of $Q$ and a system of trivialization $\sigma_{\infty}^{\alpha}$ such that

$$
A_{\infty}^{\alpha}=\sigma_{\infty}^{\alpha} D_{i}\left(\sigma_{\infty}^{\alpha}\right)^{-1}-d
$$

where $d$ denotes the exterior differentiation.

Remark IV.1. In other words we prove that the strong convergence of coulomb gauges of connections in $W^{1, n / 2}$ preserves the topology of the bundle. It was well known (see [6]) that weak convergence of connections in $W^{1, n / 2}$ does not preserve the topology of the bundle. It is straightforward to check that strong convergences of connections in $W^{1, p}$ for $p>n / 2$ preserves the topology. It is well known that strong convergence in $W^{1, p}$ for $p<n / 2$ does not preserve the topology of the bundle (Ex: the bubbling phenomenon for ASD connection in 4-dimension). So the theorem above solves a borderline case. 
Proof of Theorem IV.1. Denote $g_{i}^{\alpha, \beta}$ the sequences of transition function from $U_{\alpha} \cap U_{\beta}$ into $G$. The transition functions solve

$$
d g_{i}^{\alpha, \beta}=g_{i}^{\alpha, \beta} A_{i}^{\alpha}-A_{i}^{\beta} g_{i}^{\alpha, \beta} .
$$

From Sobolev embedding we deduce that

$$
\int_{\mathcal{U}^{\alpha, \beta}}\left|d g_{i}^{\alpha, \beta}\right|^{n} \leq C
$$

where $\mathcal{U}^{\alpha, \beta}=\mathcal{U}^{\alpha} \cap \mathcal{U}^{\beta}$. Let $p<n$, for any $i, j \in \mathbb{N}$ we have

$$
\begin{aligned}
& \int_{\mathcal{U}^{\alpha, \beta}}\left|d g_{i}^{\alpha, \beta}-d g_{j}^{\alpha, \beta}\right|^{p} \leq \int_{\mathcal{U}^{\alpha, \beta}}\left|A_{i}^{\alpha}-A_{j}^{\alpha}\right|^{p}+\int_{\mathcal{U}^{\alpha, \beta}}\left|A_{i}^{\beta}-A_{j}^{\beta}\right|^{p} \\
& +\left(\int_{\mathcal{U}^{\alpha, \beta}}\left|A_{i}^{\alpha}\right|^{n}+\left|A_{i}^{\beta}\right|^{n}+\left|A_{j}^{\alpha}\right|^{n}+\left|A_{j}^{\beta}\right|^{n}\right)^{\frac{p}{n}}\left(\int_{\mathcal{U}^{\alpha, \beta}}\left|g_{i}^{\alpha, \beta}-g_{j}^{\alpha, \beta}\right|^{\frac{n p}{n-p}}\right)^{\frac{n-p}{n}} .
\end{aligned}
$$

From (IV.2) there exists a subsequence (still denoted $g_{i}^{\alpha, \beta}$ ) such that $g_{i}^{\alpha, \beta} \rightarrow$ $g_{\infty}^{\alpha, \beta}$ in $L^{q}\left(\mathcal{U}^{\alpha, \beta}\right)$ for any $q<+\infty$, thus from (IV.3) we deduce that $g_{i}^{\alpha, \beta} \rightarrow$ $g_{\infty}^{\alpha, \beta}$ in $W^{1, p}\left(\mathcal{U}^{\alpha, \beta}\right)$ for any $p<n$. Consider a strict subcover of $\mathcal{U}^{\alpha}$ denoted $\mathcal{V}^{\alpha}$ with $\overline{\mathcal{V}^{\alpha}} \cap \overline{\mathcal{V}^{\beta}} \subset \mathcal{U}^{\alpha, \beta}$. Let $\delta>0$, there exists $\rho_{\delta}$ such that for any $\rho<\rho_{\delta}$, $\max _{z, \alpha}\left\|A^{\alpha}\right\|_{W^{1, n / 2}\left(B_{\rho}(z)\right)} \leq\left(\frac{\delta}{2}\right)^{1 / 2}$. We can cover $\mathcal{V}^{\alpha, \beta}$ by a fixed union of balls $B_{\rho}(z)$ included in $\mathcal{U}^{\alpha, \beta}$ such that $\rho<\rho_{\delta}$ and such that, applying Fubini Theorem, modulo extraction of a subsequence

$$
\int_{\partial B_{\rho}(z)}\left|\nabla\left(g_{i}^{\alpha, \beta}-g_{\infty}^{\alpha, \beta}\right)\right|^{n-\frac{1}{2}} \longrightarrow 0 .
$$

On any such a $B_{2 \rho}(z)$ from (IV.1) and the Coulomb gauge condition we have

$$
-\Delta g_{i}^{\alpha, \beta}=*\left[d g_{i}^{\alpha, \beta} \wedge * A_{i}^{\alpha}\right]+*\left[* A_{i}^{\beta} \wedge d g_{i}^{\alpha, \beta}\right] .
$$

Since $g_{i}^{\alpha, \beta} \in W^{2, n / 2}\left(\mathcal{U}^{\alpha, b e t a}\right)$ (from (IV.1)) using the Peetre-Lorentz-Sobolev embedding presented in [18] Theorem 8, we have $g_{i}^{\alpha, \beta} \in L^{n, n / 2}$. Using now the multiplication property of functions in Lorentz Spaces given in [18]: the product of 2 functions in $L^{n, n / 2}$ is in $L^{n / 2, n / 4}$. Thus, from (IV.5) again, we deduce that $\Delta g_{i}^{\alpha, \beta}$ is bounded in $L^{n / 2, n / 4}\left(B_{2 \rho}(z)\right)$, reducing a bit the size of the ball (from $2 \rho$ to $3 \rho / 2$ ), using the weak $(r, r)$ property of Riesz operator and Theorem 3.15 of Chap. $\mathrm{V}$ of [17], we have that $\left\|\nabla^{2} g_{i}^{\alpha, \beta}\right\|_{L^{n / 2, n / 4}}$ is 
bounded independently on $i$. Bootstraping this information again in (IV.5) we get that $\Delta g_{i}^{\alpha, \beta}$ is bounded in $L^{n / 2, n / 6}\left(B_{2 \rho}(z)\right) \ldots$ and so on untill the second exponent reaches $\frac{n}{n-2}$. So we deduce that

$$
\left\|\nabla g_{i}^{\alpha, \beta}\right\|_{L^{n, \frac{n}{n-2}}\left(B_{\rho}(z)\right)} \leq C
$$

where $C$ is independent on $i$. We have (all norms above being taken over $\left.B_{\rho}(z)\right)$

$$
\begin{aligned}
& \left\|\Delta\left(g_{i}^{\alpha, \beta}-g_{\infty}^{\alpha, \beta}\right)\right\|_{L^{\frac{n}{2}, 1}} \\
& \leq\left\|\nabla\left(g_{i}^{\alpha, \beta}-g_{\infty}^{\alpha, \beta}\right)\right\|_{L^{n, n / n-2}}\left[\left\|A_{i}^{\alpha}\right\|_{L^{n, \frac{n}{2}}\left(B_{\rho}(z)\right)}+\left\|A_{i}^{\beta}\right\|_{L^{n, \frac{n}{2}}}\right] \\
& +\left[\left\|A_{i}^{\alpha}-A_{\infty}^{\alpha}\right\|_{L^{n, \frac{n}{2}}}+\left\|A_{i}^{\beta}-A_{\infty}^{\beta}\right\|_{L^{n, \frac{n}{2}}}\right]\left[\left\|\nabla g_{i}^{\alpha, \beta}\right\|_{L^{n, \frac{n}{n-2}}}+\left\|\nabla g_{\infty}^{\alpha, \beta}\right\|_{L^{n, \frac{n}{n-2}}}\right] .
\end{aligned}
$$

Since we have $\int_{B_{\rho}(z)}\left|A_{i}^{\alpha}\right|^{\frac{n}{2}}+\left|\nabla A_{i}^{\alpha}\right|^{\frac{n}{2}} \leq \delta$, we deduce that $\int_{B_{\rho}(z)}\left|A_{i}^{\alpha}\right|^{n} \leq$ $\left(\delta^{2 / n}+\rho^{n-2} \delta^{2 / n}\right)^{n} \leq C \delta^{2}$ where $C$ is a universal constant (we may allways assume that $\rho<1)$. Using the embedding of $W^{1, n / 2}\left(B_{\rho}\right)$ into $L^{n, n / 2}\left(B_{\rho}\right)$, taking into account the two previous estimates and the common scaling behavior of the norms $L^{n}, L^{n, n / 2}$ and the pseudo-norm: gradient in $L^{n / 2}$ in n dimension, we have

$$
\left\|A_{i}^{\alpha}\right\|_{L^{n, n / 2}\left(B_{\rho}\right)} \leq C \delta^{2}
$$

Let $h$ solving

$$
\begin{cases}\Delta h=\Delta\left(g_{i}^{\alpha, \beta}-g_{\infty}^{\alpha, \beta}\right) & \text { in } B_{\rho}(z) \\ h=0 & \text { on } \partial B_{\rho}(z) .\end{cases}
$$

Standard result on Riesz Kernel gives that the operator that assign $\nabla^{2} h$ to $\Delta\left(g_{i}^{\alpha, \beta}-g_{\infty}^{\alpha, \beta}\right)$ is a weak $(r, r)$ operator for $r$ between 0 and $\infty$. Thus we have

$$
\begin{aligned}
\|\nabla h\|_{L^{n, \frac{n}{n-2}\left(B_{\rho}\right)}} & \leq\|\nabla h\|_{L^{n, 1}\left(B_{\rho}\right)}+\left\|\nabla^{2} h\right\|_{L^{n / 2,1}\left(B_{\rho}\right)} \\
& \leq C\left\|\Delta\left(g_{i}^{\alpha, \beta}-g_{\infty}^{\alpha, \beta}\right)\right\|_{L^{n / 2,1}\left(B_{\rho}\right)}
\end{aligned}
$$


where $C$ is independent on $\rho$. Let now $k$ solving

$$
\begin{cases}\Delta k=0 & \text { in } B_{\rho}(z) \\ k=g_{i}^{\alpha, \beta}-g_{\infty}^{\alpha, \beta} & \text { on } \partial B_{\rho}(z) .\end{cases}
$$

From standard elliptic estimates we have

$$
\|k\|_{W^{1-\frac{1}{s}, s}\left(\partial B_{\rho}(z)\right)} \leq C\left\|g_{i}^{\alpha, \beta}-g_{\infty}^{\alpha, \beta}\right\|_{W^{1, n-\frac{1}{2}}\left(\partial B_{\rho}(z)\right)}
$$

for $s=n\left(\frac{2 n-1}{2(n-1)}\right)>n$. Thus we deduce

$$
\|\nabla k\|_{L^{n, \frac{n}{n-2}}\left(B_{\rho}(z)\right)} \leq C_{\rho}\left\|g_{i}^{\alpha, \beta}-g_{\infty}^{\alpha, \beta}\right\|_{W^{1, n-\frac{1}{2}}\left(\partial B_{\rho}(z)\right)} .
$$

Combining (IV.7)...(IV.13)

$$
\begin{aligned}
& \left\|\nabla\left(g_{i}^{\alpha, \beta}-g_{\infty}^{\alpha, \beta}\right)\right\|_{L^{n, \frac{n}{n-2}}\left(B_{\rho}(z)\right)} \\
& \leq C \delta^{2}\left\|\nabla\left(g_{i}^{\alpha, \beta}-g_{\infty}^{\alpha, \beta}\right)\right\|_{L^{n, \frac{n}{n-2}}\left(B_{\rho}(z)\right)} \\
& \quad+C\left[\left\|A_{i}^{\alpha}-A_{\infty}^{\alpha}\right\|_{L^{n, n / 2}\left(B_{\rho}(z)\right)}+\left\|A_{i}^{\beta}-A_{\infty}^{\beta}\right\|_{L^{n, n / 2}\left(B_{\rho}(z)\right)}\right] \\
& \quad+C_{\rho}\left\|g_{i}^{\alpha, \beta}-g_{\infty}^{\alpha, \beta}\right\|_{W^{1, n-\frac{1}{2}}\left(\partial B_{\rho}(z)\right)}
\end{aligned}
$$

and from (IV.4) and the embedding of $W^{1, n / 2}$ into $L^{n, n / 2}$ we deduce that $A_{i}^{\alpha} \rightarrow A_{\infty}^{\alpha}$ in $L^{n, \frac{n}{n-2}}$, thus

$$
\left\|\nabla\left(g_{i}^{\alpha, \beta}-g_{\infty}^{\alpha, \beta}\right)\right\|_{L^{n, \frac{n}{n-2}}\left(B_{\rho}(z)\right)} \longrightarrow 0 .
$$

Bootstraping this information into (IV.7) we get

$$
\left\|\Delta\left(g_{i}^{\alpha, \beta}-g_{\infty}^{\alpha, \beta}\right)\right\|_{L^{n / 2,1}\left(B_{\rho}(z)\right)} \longrightarrow 0
$$

thus for any $\lambda<1$ we have, using again the weak $(r, r)$ property of the Riesz operator,

$$
\left\|\nabla^{2}\left(g_{i}^{\alpha, \beta}-g_{\infty}^{\alpha, \beta}\right)\right\|_{L^{n / 2,1}\left(B_{\lambda \rho}(z)\right)} \longrightarrow 0 .
$$

Using now a Lorentz-Sobolev embedding (Theorem 8 [18]) we deduce

$$
\left\|\nabla\left(g_{i}^{\alpha, \beta}-g_{\infty}^{\alpha, \beta}\right)\right\|_{L^{n, 1}\left(B_{\lambda \rho}(z)\right)} \longrightarrow 0 .
$$


At this stage of the proof we are going to use the key embedding established by $\mathrm{H}$. Brezis and S. Waigner [3] which says that a function on $\mathbb{R}^{n}$ whose derivatives are in $L^{n, 1}$ is in $L^{\infty}$. This can be shown in the following way: assume $\nabla f \in L^{n, 1}\left(\mathbb{R}^{n}\right)$, we have $f(x)=\operatorname{div} \int_{\mathbb{R}^{n}} G(x-y) f(y)$ where $G$ is the Kernel of the Laplacian on $\mathbb{R}^{n}$. Then since $|\nabla G(x)| \leq C /|x|^{n-1}$ we have $|\{x ;|\nabla G|(x) \geq \lambda\}| \leq C / \lambda^{-n / n-1}$ which means that the weak $L^{n / n-1}$-norm of the derivatives of $G$ is bounded: $\|\nabla G\|_{L^{\frac{n}{n-1}, \infty}\left(\mathbb{R}^{n}\right)} \leq C$. The claim follows then from the duality $L^{n, 1}-L^{\frac{n}{n-1}, \infty}$. Thus, granting this embedding and inequality (IV.17), we deduce, since the balls $B_{\rho}$ cover $\mathcal{V}^{\alpha} \cap \mathcal{V}^{\beta}$,

$$
\left\|g_{i}^{\alpha, \beta}-g_{\infty}^{\alpha, \beta}\right\|_{L^{\infty}\left(\mathcal{V}^{\alpha} \cap \mathcal{V}^{\beta}\right)} \rightarrow 0 .
$$

Using now Proposition 3.2 of [21] we deduce that, for $i$ large enough, $g_{i}^{\alpha, \beta}$ and $g_{\infty}^{\alpha, \beta}$ are equivalent cocycle for the Cech cohomology (see [4]), in other words there exist trivialisations $\rho^{\alpha}$ over sets (eventually slightly smaller than the $\left.\mathcal{V}^{\alpha}\right)$ such that $\left(g_{i}^{\alpha, \beta}\right)^{-1} g_{\infty}^{\alpha, \beta}=\rho^{\alpha}\left(\rho^{\beta}\right)^{-1}$. The equivalence of the cocycles imply that they define toplogically the same bundles and Theorem IV.1 is proved.

If we do not assume anymore that we are working with Coulomb gauges we have the following result that generalises the previous theorem.

Theorem IV.2. Let $Q$ be a principal G-bundle over a compact $n$ dimensionnal riemannian manifold $M$. Assume there exists on $Q$ a sequence of connections $D_{i}$, a fixed covering of $M\left(\mathcal{U}^{\alpha}\right)$ and a trivialization $\sigma_{i}^{\alpha}$ over $\mathcal{U}^{\alpha}$ such that if $A_{i}^{\alpha}=\sigma_{i}^{\alpha} D_{i}\left(\sigma_{i}^{\alpha}\right)^{-1}-d$ converges strongly to some $A_{\infty}^{\alpha}$ in $W^{1, n / 2}$ and if $d^{*} A_{i}^{\alpha}$ strongly converges to $d^{*} A_{\infty}^{\alpha}$ in the Lorentz space $L^{n / 2,1}$, then $A_{\infty}^{\alpha}$ defines a connection of the same bundle $Q$ : there exists a connection $D_{\infty}$ of $Q$ and a system of trivialization $\sigma_{\infty}^{\alpha}$ such that

$$
A_{\infty}^{\alpha}=\sigma_{\infty}^{\alpha} D_{i}\left(\sigma_{\infty}^{\alpha}\right)^{-1}-d
$$

where $d$ denotes the exterior differentiation.

Remark IV.2. In the particular cases where the topology of the bundle is given by the second Chern class only, for instance for $G=S U(2)$ over an orientable 4-manifold, the result above does not require anymore $d^{*} A_{i}$ to converge in $L^{2,1}$ but only in $L^{2,2}$ (we only assume that $A_{i}$ converges in $\left.W^{1,2}\right)$. This is a consequence of the integral formula for the Chern class (see [22]). To know whether the strong $L^{n / 2,1}$ convergence of $d^{*} A_{i}^{\alpha}$ is really needed in the statement of Theorem IV.2, remains an open question. 
Proof of Theorem IV.2. The goal is to find a change of trivialisation in which we fulfill the assumption of Theorem IV.1: strong $W^{1, n / 2}$ convergence of the new $A_{i}^{\alpha}$ that will be Coulomb $d^{*} A_{i}^{\alpha}=0$.

We can assume that the $\mathcal{U}^{\alpha}$ are diffeomorphic to $B^{n}$ and that the $W^{1, n / 2}$ norm of $A_{\infty}^{\alpha}$ on $\mathcal{U}^{\alpha}$ is bellow the range $\kappa$ where Uhlenbeck's theorems in [21] apply. First of all we show that we can assume that $d^{*} A_{i}^{\alpha} \rightarrow 0$ in $L^{n / 2,1}\left(\mathcal{U}^{\alpha}\right)$. Indeed let $\sigma^{\alpha}$ be the change of gauge for $A_{\infty}^{\alpha}$ given by Corollary 2.2 in [21]: we have that $\hat{A}_{\infty}^{\alpha}=\left(\sigma^{\alpha}\right)^{-1} A_{\infty}^{\alpha} \sigma^{\alpha}+\left(\sigma^{\alpha}\right)^{-1} d \sigma^{\alpha}$ verifies

$$
d^{*} \hat{A}_{\infty}^{\alpha}=0 \quad \text { and } \quad\left\|\hat{A}_{\infty}^{\alpha}\right\|_{W^{1, n / 2}\left(\mathcal{U}^{\alpha}\right)} \leq C \kappa
$$

since $d^{*} \hat{A}_{\infty}^{\alpha}=0, \sigma^{\alpha}$ solves

$$
\Delta \sigma^{\alpha}=*\left[d \sigma^{\alpha} \wedge * \hat{A}_{\infty}^{\alpha}\right]-d^{*} A_{\infty}^{\alpha} \sigma^{\alpha}+*\left[* A_{\infty}^{\alpha} \wedge d \sigma^{\alpha}\right] .
$$

Argueing as in the proof of the previous theorem, we have $\left\|A_{\infty}^{\alpha}\right\|_{L^{n, n / 2}} \leq$ $C,\left\|\hat{A}_{\infty}^{\alpha}\right\|_{L^{n, n / 2}} \leq C$ and thus $\left\|d \sigma^{\alpha}\right\|_{L^{n, n / 2}} \leq C$, using the hypothesis of the theorem and multiplication properties in the Lorentz spaces mentioned above we have

$$
\left\|\Delta \sigma^{\alpha}\right\|_{L^{n / 2, n / 4}\left(\mathcal{U}^{\alpha}\right)} \leq C .
$$

Using the weak $(r, r)$ property of the Riesz operator and Theorem 3.15 Chapter V of [17], we deduce that for any $\omega \subset \subset \mathcal{U}^{\alpha}$

$$
\left\|\nabla^{2} \sigma^{\alpha}\right\|_{L^{n / 2, n / 4}(\omega)} \leq C .
$$

Having taken a slightly larger ball than $\mathcal{U}^{\alpha}$ from the begining, we may always assume that the inequality above holds for $\omega=\mathcal{U}^{\alpha}$. Then we deduce that $\left\|n a b l a \sigma^{\alpha}\right\|_{L^{n, n / 4}\left(\mathcal{U}^{\alpha}\right)} \leq C$ and as in the proof of Theorem IV.1 we keep bootstraping this information in (IV.19) untill reaching

$$
\left\|\nabla^{2} \sigma^{\alpha}\right\|_{L^{n / 2,1}\left(\mathcal{U}^{\alpha}\right)} \leq C .
$$

We then proceed to the change of gauge given by $\sigma^{\alpha}$ for the complete sequence $A_{i}^{\alpha}$ and we denote $\hat{A}_{i}^{\alpha}=\left(\sigma^{\alpha}\right)^{-1} A_{i}^{\alpha} \sigma^{\alpha}+\left(\sigma^{\alpha}\right)^{-1} d \sigma^{\alpha}$ clearly we have that the hypothesis of the theorem are preserved and that $d^{*} \hat{A}_{i}^{\alpha} \rightarrow 0$ in $L^{n, 1}$. At this stage of the proof we will need the corresponding lemma to Lemma 2.7 of [21] but with Lorentz norms: Denote

$$
\begin{gathered}
\tilde{W}_{1}^{1, n / 2}\left(\wedge^{1} B^{n}, \mathfrak{G}\right)=\left\{a \in W^{1, n / 2}\left(\wedge^{1} B^{n}, \mathfrak{G}\right) ; d^{*} a \in L^{n / 2,1}\right\}, \\
W_{1,0}^{2, n / 2}\left(B^{n}, G\right)=\left\{s \in W^{2, n / 2}\left(B^{n}, G\right) ; \nabla^{2} s \in L^{n / 2,1} \text { and } s=\text { id on } \partial B^{n}\right\} .
\end{gathered}
$$

Using the above notations we have 
Lemma IV.1. There exists $\delta>0$ such that if $A$ in $W^{1, n / 2}\left(\wedge^{1} B^{n} ; \mathfrak{G}\right)$ verifying $d^{*} A=0$ and $\|A\|_{W^{1, n / 2}} \leq \delta$, then there exists $\varepsilon>0$ such that for any $a \in \tilde{W}_{1, \nu}^{1, n / 2}\left(\wedge^{1} B^{n}, \mathfrak{G}\right)$ verifying $\|a\|_{\tilde{W}_{1}^{1,2}} \leq \varepsilon$, the non linear equation

$$
d^{*}\left(s^{-1} d s+s^{-1}(A+a) s\right)=0
$$

has a solution $s$ in $W_{1,0}^{2, n / 2}\left(B^{n}, G\right)$ that depends smoothly on $a \in \tilde{W}_{1}^{1, n / 2}$.

Proof of Lemma IV.1. Consider the spaces

$$
\hat{W}_{1,0}^{2, n / 2}\left(B^{n}, \mathfrak{G}\right)=\left\{U \in W^{2, n / 2}\left(B^{n}, G\right) ; \nabla^{2} U \in L^{n / 2,1}, U=0 \text { on } \partial B^{n}\right\} .
$$

Using Brezis-Wainger-Sobolev embedding we have

$$
\|U\|_{L^{\infty}\left(B^{n}\right)} \leq C\|U\|_{\hat{W}_{1,0}^{2, n / 2}\left(B^{n}\right)} .
$$

This is the key point that permits to adapt Uhlenbeck arguments to the present situation and the $L^{n / 2,1}$ assumption on the second derivatives is crucial at this stage. From (IV.23) we deduce that the maps $U \rightarrow \exp (U)$ or $\exp (-U)$ from $\hat{W}_{1,0}^{2, n / 2}$ into $L^{\infty}$ are smooth. Thus the map

$$
\begin{aligned}
& \hat{W}_{1,0}^{2, n / 2} \otimes \tilde{W}_{1}^{1, n / 2} \rightarrow L^{n / 2,1} \\
& (U, a) \longrightarrow d^{*}[\exp (-U) d \exp (U)+\exp (-U)(A+a) \exp (U)]
\end{aligned}
$$

is smooth (we also use that $d^{*} A=0$, and again that for $U$ in $\hat{W}_{1,0}^{2, n / 2}$ and $a$ in $\tilde{W}_{1}^{1, n / 2}, d U \in L^{n, \frac{n}{n-2}}$ and $a \in L^{n, n / 2}$ too - we even have $\left.d U \in L^{n, 1}\right)$. At $(U, \lambda)=(0,0)$ we consider the linearized operator

$$
H(\psi)=d^{*} d \psi+*([* A, d \psi]) .
$$

Because of the boundary condition $U=0$ on $\partial B^{n}, d^{*} d \psi=\Delta \psi$ is invertible from $\hat{W}_{1,0}^{2, n / 2}$ into $L^{n / 2,1}$, where we use again that Riesz operator is weak $(r, r)$ and Theorem 3.15 Chapter V of [17]. Thus we have

$$
\|H(\psi)\|_{L^{n / 2,1}} \geq C_{1}\|\psi\|_{\hat{W}_{1,0}^{2,2}}-C_{2}\|A\|_{L^{n, n / 2}}\|\psi\|_{\hat{W}_{1,0}^{2,2}}
$$

Thus choosing $\|A\|_{W^{1, n / 2}}$ small enough makes $\|A\|_{L^{n, n / 2}}$ small, which makes $H$ invertible and applying the local inversion theorem we establish Lemma IV.1. 
End of the proof of Theorem IV.2. We can apply Lemma IV.18 above to $A=$ $\hat{A}_{\infty}^{\alpha}$ and $a=\hat{A}_{i}^{\alpha}-\hat{A}_{\infty}^{\alpha}$ for $i$ large enough such that $\left\|\hat{A}_{i}^{\alpha}-\hat{A}_{\infty}^{\alpha}\right\|_{\tilde{W}_{1}^{1, n / 2}} \leq \varepsilon$ and we generate a last change of gauge that put us in the setting of Theorem IV.1 above that we can apply and Theorem IV.2 is proved.

\section{References.}

[1] W. Allard, An integrality theorem and a regularity theorem for surfaces whose first variation with respect to a parametric elliptic integrand is controlled, Proc. Symp. Pure Math., 44 (1986), 1-28.

[2] H. Brezis, Laser beams and limiting cases of Sobolev inequalities, Non linear P.D.E., College de France Seminar, II, H. Brezis \& J.L. Lions, eds., Pitman, 1982.

[3] H. Brezis and S. Wainger, A note on limiting cases of Sobolev embeddings and convolution inequalities, Comm. P.D.E., 5 (1980), 773-789.

[4] R. Bott and L. Tu, Differential forms in Algebraic Topology, Springer, 1982.

[5] W.Y. Ding and G. Tian, Energy identity for a class of approximate harmonic maps from surfaces, Comm. Anal. Geom., 3 (1995), 543-554.

[6] S.K. Donaldson and P.B. Kronheimer, The geometry of Four-Manifolds, Oxford, 1990.

[7] S.K. Donaldson and R.P. Thomas, Gauge Theory in higher dimensions, in 'The geometric Universe' (Oxford, 1996), Oxford Univ. Press, (1998), $31-47$.

[8] F. Hélein, Harmonic maps, Conservation laws and moving frames, Diderot, 1996.

[9] F.H. Lin, Gradient estimates and blow-up analysis for stationary harmonic maps, Ann. Math., 149 (1999), 785-829.

[10] F.H. Lin and T. Rivière, A Quantization Property for Static GinzburgLandau Vortices, Comm. Pure App. Math., 54 (2001), 206-208.

[11] F.H. Lin and T. Rivière, Energy Quantization for Harmonic Maps, Duke Math. Journal, 2002. 
[12] F.H. Lin and T. Rivière, A quantization property for moving line vortices, Comm. Pure App. Math., 54 (2001), 826-850.

[13] Y. Meyer and T. Rivière, A partial regularity result for a class of stationary Yang-Mills Fields in high dimensions, to appear in Rev. Mat. Iberoamericana, 20002.

[14] H. Nakajima, Compactness of the moduli space of Yang-Mills connections in higher dimensions, J. Math. Soc. Japan, 40 (1988), 383-392.

[15] J. Peetre, Espaces d'interpolation et théorème de Soboleff, Ann. Inst. Fourier, Grenoble, 16 (1966), 279-317.

[16] T. Parker, Bubble tree convergence for harmonic maps, J. Differential Geom., 44 (1996), 545-633.

[17] E. Stein and G. Weiss, Introduction to Fourier analysis on Euclidian spaces, Princeton Univ. Press, 1971.

[18] L. Tartar, Imbedding Theorems of Sobolev Spaces into Lorentz Spaces, Boll. U.M.I., $1 B$ (1998) 479-500.

[19] G. Tian, Gauge theory and calibrated geometry, I, Ann. Math., 151 (2000), 193-268.

[20] K. Uhlenbeck, Removable singularities in Yang-Mills fields, Comm. Math. Phys., 83 (1982), 11-29.

[21] K.Uhlenbeck, Connections with $L^{p}$ bounds on curvature, Comm. Math. Phys., 83 (1982), 31-42.

[22] K.Uhlenbeck, The Chern classes of Sobolev connections, Comm. Math. Phys., 101 (1985), 449-457.

D-MATH

ETH-ZENTRUM

8092 ZÜRICH

SWITZERLAND

RECEIVED DECEMBer 12, 2000. 